We estimated the difference between potential savings if the adjustment had been perfect and the actual saving obtained $(€ 21,135)$, possibly caused by the preparation process or expiry of some reconstituted vials

Conclusions Residues management is a common practise to improve the efficiency of the preparation process. Optimizing this process of updating medicines' stabilities, recording the opening date on the vial, checking expiries and storage conditions, achieved significant savings in the cost of treatments.

No conflict of interest.

\section{OHP-057 MEASURES FOR PALIVIZUMAB COST CONTAINMENT ANALYSIS}

doi:10.1136/ejhpharm-2013-000276.431

M Bullejos Molina, J Nazco Casariego, I Rodriguez Pedrosa, J Gonzalez Garcia, I Gonzalez Perera. Hospital Universitario de Canarias, Servicio de Farmacia, La Laguna, Spain

Background Prescription RSV (Respiratory Syncytial Virus) immunoprophylaxis with palivizumab involves high pharmaceutical costs associated with paediatric services. It is necessary to establish protocols aimed at reducing the cost associated with these treatments, adjusted to the best cost-effectiveness criteria.

Purpose To assess whether the prescriptions are consistent with indications of greater efficiency; to assess the impact of the revision of the criteria in the last vaccination campaign.

Materials and Methods We analysed the cost associated with the use of palivizumab in the last six years, the criteria for indication of prophylaxis, and the impact of the restrictions introduced last season. The number of doses that can be administered has been restricted: a limitation for the higher-risk months (Nov-Jan), and more cost-effective presentations $(100 \mathrm{mg})$ are to be used.

We extracted from our hospital system (SAP) the dispensed prescriptions of palivizumab from September 2006 to February 2012 (5 vaccination campaigns) analysing the number of patients treated, number of doses per child, vaccination period, consumption distribution among different presentations, indication criteria and associated cost.

Results An examination of the last 6 vaccination campaigns shows the impact of the measures taken. We obtained a $35 \%$ cost reduction $(€ 98,875.25)$ compared to the average in recent seasons, and a $28 \%$ decrease in the number of children treated. The priority of using 100 $\mathrm{mg}$ vials meant a $63 \%$ reduction in the use of $50 \mathrm{mg}$ vials, which are less cost-effective. The largest decrease $(10 \%)$ in prescriptions was in premature infants between 29 and 35 weeks gestation. No vaccinations were done in March.

Conclusions Establishing agreed more restrictive criteria used in the selection of patients to be treated, limiting the months in which the vaccine can be administered and the preferential use of $100 \mathrm{mg}$ vials has brought about a $35 \%$ reduction in the cost associated with this treatment $(€ 98,875.25)$ compared to previous campaigns.

No conflict of interest.

\section{OHP-058 NEW RESPONSIBILITIES FOR PHARMACY TECHNICIANS: THE SKILLS MATRIX, A PERFECT TOOL FOR CHANGE MANAGEMENT}

doi:10.1136/ejhpharm-2013-000276.432

E Barbier, L Halcewicz, S Esposito, A Rieutord, S Barbault-Foucher. Hôpital Antoine Béclère, Pharmacy, Clamart, France

Background Our teaching hospital has a level 3 maternity unit and a neonatal intensive care unit for 29 preterm infants. Over 3,000 bags of paediatric parenteral nutrition are prescribed annually. Their production is outsourced to another hospital. Until 2010, only pharmacy residents and pharmacists were in charge of this activity.

Purpose To design and implement a skills matrix to shift this activity towards the hospital pharmacy technicians.

Materials and Methods A multidisciplinary working group (a pharmacist, a chief technician, a pharmacy resident, two pharmacist technicians (PTs)) defined Standard Operating Procedures (SOPs) needed and skill levels according to our Process Map. They established a training programme and finally a Gantt chart.

Results Our matrix includes two levels: level 2 consists of the delivery of parenteral nutrition; level 1 also includes ordering and checking nutrition bags, the management of nonconforming products and monthly management.

Of the 11 pharmacy technicians, 100\% gained level 2 and 55\% level 1 between January and May 2011 as defined. The activity shift was fully completed after 6 months. SOPs were reviewed and approved entirely during 2011. Experience feedback meetings have been set on a regular basis with the clinical ward to maintain standards since June 2012.

Pharmacy technicians have expanded their skills and this has enabled us to save pharmacists' time (0.3 Full Time Equivalent). PTs were examined again in September 2012 in order to assess their skills and knowledge after one year, using interactive real-life exercises.

Conclusions The skills matrix is a simple and attractive management tool for identifying needs, assessing and developing individual skills. It provides not only a clear insight into individual skills but also into transversal competencies in a Pharmacy Department. It is particularly adapted to conducting change in a peaceful and positive manner and very important for annual individual assessment.

No conflict of interest.

\section{OHP-059 OPTIMIZATION OF HIGH-IMPACT MEDICINES IN PAEDIATRICS}

doi:10.1136/ejhpharm-2013-000276.433

R Tamayo Bermejo, C Gallego Fernández, J González Chávez, M Ruiz de Villegas, I Muñoz Castillo. HRU CARLOS HAYA, Pharmacy, Málaga, Spain

Background High economic impact medicines are used off-label in paediatric situations, using adult presentations for lack of a paediatric form.

Purpose To justify preparing individualised medicines for paediatric use according to individual need; adaptation to increase safety and reduce costs.

Materials and Methods Retrospective review of high-impact medicines used in individualised treatment in paediatrics. Duration of study: 4 years. The medicines were included if they had been needed (adalimumab 35 months, anakinra 73 months and pegfilgrastim 50 months).

Data collection sources: Computer application in the pharmacotechnical area, software of the outpatient dispensing and management system. Personnel times were collected according to the Catalogue of Products and Billing (2nd edition 2009) and costs according to the Analytical Accounting Service. As these were standard sterile formulas the time and cost of pharmaceutical personnel were considered (standard operating procedure of a new product and successive validations), nurse (production) and technician (material preparation, labelling and packaging.

We compared the cost of dispensing the complete pharmaceutical form with individualised costs through sterile repackaging

Variables studied: patients, different types of dosages, number of syringes made, number of syringes consumed and associated costs. For economic valuation the cost of the commercial presentation and the personnel involved in the making were considered.

Results The 3 medicines identified were repacked from the adult branded product formulations. 